\title{
Research on the None Mercury Electrode Electrochemical and the Core Applications on Waste Water Metal Ions Detection under the Background of Green Industry
}

\author{
Juan Yang \\ Wuchang ShouYi University, \\ Wuhan, Hubei, 430064 China
}

\begin{abstract}
In this paper, we conduct research on none-mercury electrode electrochemical and the core applications on waste water metal ions detection under the background of green industry. The excess consumption of natural resources and ecological environment of serious imbalance that has aroused worldwide for keeping a close eye on environment protection with fundamental shift 21 st century industrial design concept, environment consciousness of industrial design has become the dominant consciousness, and "green design" is the requirement of industrial design in saving energy, recycling, improve efficiency, etc. more conducive to the protection of the environment. Therefore, advocating "green design" has become a new design industrial design guidelines. With this historical background we propose the novel waste water metal ions detection method that is meaningful.
\end{abstract}

Keywords- None Mercury, Electrode Electrochemical, Waste Water, Metal Ions, Green Industry.

\section{Introduction}

Before further elaboration, I think it is necessary for everyone to introduce the related, first of all, we know that there are many existing in the contemporary society on the basic energy and environmental problems, these problems at the time of the increasingly prominent, but behind it all reflects the human in the process of its long-term development of the result of the abuse and exploitation, directly led to the conflict of the human and the environment. So as we described above, we must make some measures to maintain normal, healthy development, therefore, green chemistry act as a very important role in among them, we need to do is through the green chemistry engineering and technology, reduce the input of some of the damage to the environment in the process of reaction of reactant, and try to produce some for the human and environment friendly products. We can always seek some way in the process of improvement to reduce the harmful reactants and products, and increase the beneficial products which is advocated by the green chemistry in the process of its development, and also is the so-called man and nature lives in the harmony condition [1-3].

From a technical perspective the development of human society can be divided into the traditional technology, gray and green technology which aims to improve green technology era for the human production and social activities and destruction of basic natural environment, is to realize the human sustainable development to protect the environment, maintain the ecological balance of the sum total of all kinds of the general means. Widely in the social production practices, green design its profound significance in following aspects. (1) Promote technological progress and technological innovation. Green design requirements at the same time, considering the social, economic and ecological benefits, it puts forward higher requirements on the design of the product, so as to promote the technological progress and innovation, meet the needs of human social and economic sustainable development. (2) To meet the requirements of 
international trade. Based on the idea of the green design is to create the products can better adapt to the international standard, that make our country a wider range of green products can enter the international market, enhance competitiveness of products in the international market. (3) The important measures to meet the knowledge economy era. Developing green design ideas under the guidance of the green industry's largest significance in environmental protection in the coming of knowledge economy in the $21 \mathrm{st}$ century, green design, green products and green industry will be an important aspect of enterprise competition and it is to coordinate the relationship between population, resources, environment and development in our country is important one annulus.
Sustainable development is more and more important in today's society, therefore in the production of chemical industry should follow the guiding thoughts, uses the high selectivity of raw materials, energy conservation and emissions reduction, using high-tech chemical catalyst, the greatest degree of reducing emissions and increasing the effective product purity, on the premise of limited resources, protect the ecological environment, maintain the ecological balance of existing. Green chemistry in the development of the whole chemical industry has the substantial meaning, the use of high and new technical products catalyst can change the existing industrial structure and the traditional production process to accelerate the development of chemical industry [4-5].

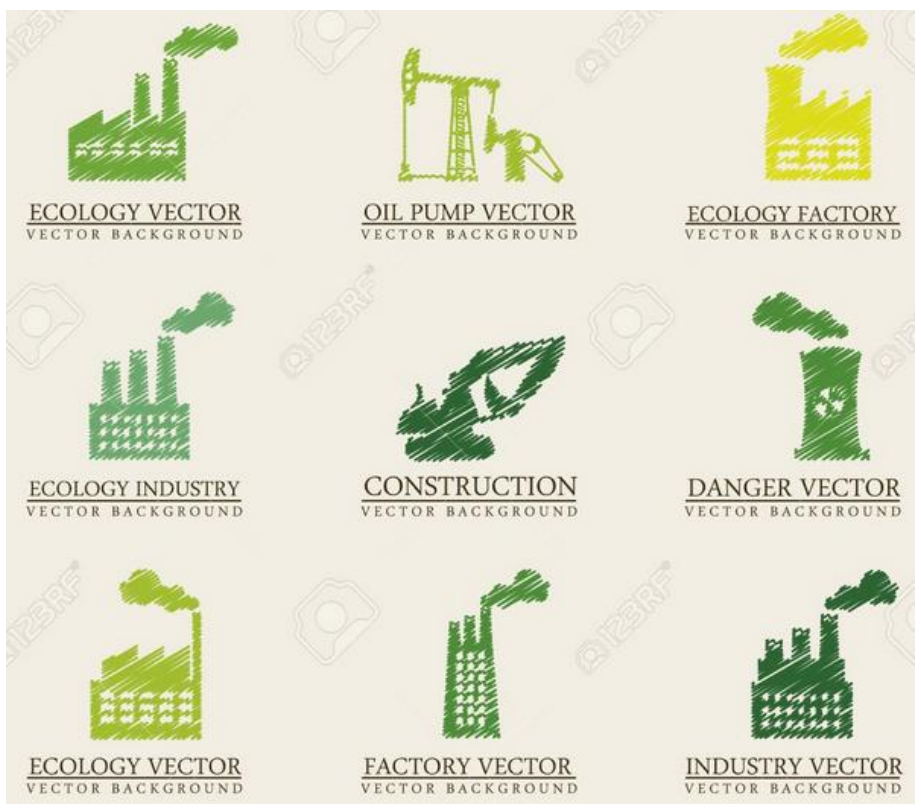

Figure 1. The Demonstration of Scenarios of the Green Industry Applications

In this paper, we conduct research on the none-mercury electrode electrochemical and the core applications on waste water metal ions detection under the background of green industry. We can put the definition of non-pollution chemistry, so in the process of general green chemistry technology was produced by certain means is green chemical industry technology, using its principle from the root to the destruction of the ordinary chemical reaction to produce. In the following sub-sections, we will conduct the corresponding research with the assistance of the literature reviews.

\section{The Proposed Perspective}

The Principles of the Green Chemistry. Green chemistry is to use chemical technology and method to avoid or reduce the harmful to the environment of the general raw materials, catalysts, solvents and reagents used in the 
production process at the same time in the production process does not produce toxic or harmful by-products that holds the following advantages.

- Way different from the traditional chemical industry, that mainly adopts the green chemical engineering are green pollution-free raw materials, and can be used at the same time, give full play to the value of raw materials, ensure no damage to the environment, reduce the economic pressure, and save the corresponding energy.

- The traditional chemicals are extracted from the raw materials to use of material resources, resources waste problem. And green chemical research can successfully avoid the happening of the problem. It effectively change the molecular structure of matter, and leave the material characteristics, converting useless material to can make full use of resources, at this point can effectively solve the problem of resources waste.

People in the pursuit of the high-tech development at the same time, also began to emphasize the protection of environment, more and more pay attention to the importance of environment, therefore, in the development of general science and technology at the same time, are also committed to the implementation of green technology, the green circular economy, green products, the green chemical engineering greets people a lot of vocabulary, and especially the chemical such large damage to the environment, but also very pay attention to green. The rise of green chemical, to a certain extent, eases the problems of social environment made a certain contribution to the protection of the environment.
Green chemistry not only has the significant social, economic and environmental benefits, and the chemical itself is also an opportunity that is a new stage in the development of chemistry. From the perspective of the history of chemistry development, due to the needs of the development of science and technology and society, a lot of stages, each stage have focus, goals. Once the key, goals are social identity, there will be more personnel and material resources into the field. At the same time, due to the government, the attention of social public opinion and intelligence, information developed and rapidly, and scientific research of the organizational planning, stronger; Policy, law, more guidance; The scientific research foundation more assured; Business and academic cooperation more widely; Academic research more deeply; Science and technology education deeper and more extensive as all of these factors contribute to the realization of the goal of science and technology [6].

The Waste Metal Ions Detection. Widespread heavy metal ions in industrial wastewater, mainly from the mine pit drainage, drainage, non-ferrous metal smelter dust removal of non-ferrous metals processing factory acid water, electroplating factory plating washing water, drainage, steel pickling and electrolysis, pesticide, medicine, paint, paint and other industrial waste water. Heavy metal ion wastewater ruled out, even if the concentration is small, also can cause nuisance as has the toxic effect of heavy metal ion wastewater pollution, biological characteristics of non-biodegradable, for the long time it can cause cancer in organisms accumulate through the food chain as it has attracted attention of the great many environmental experts. 


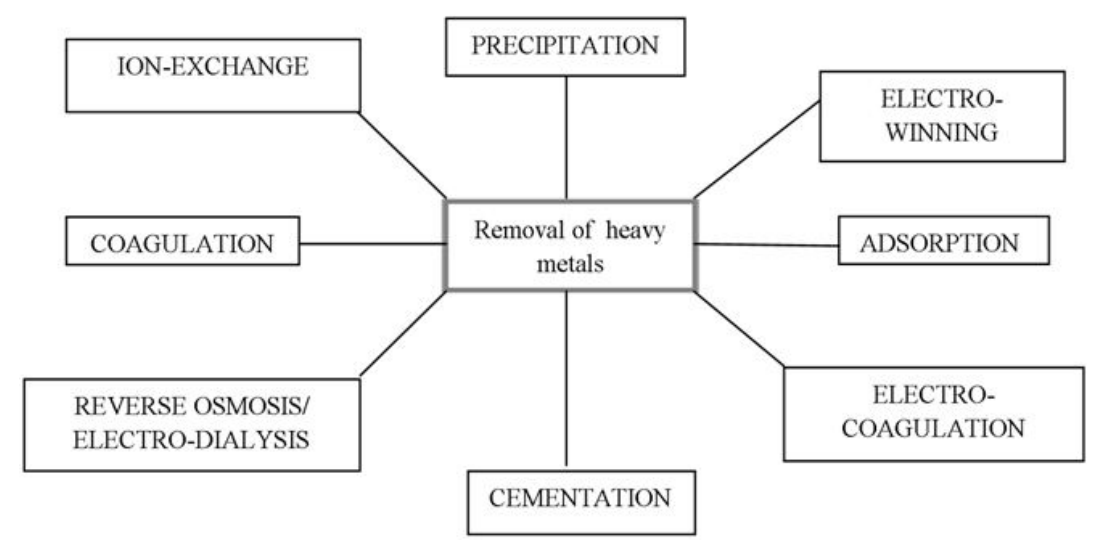

Figure 2. The Flowchart and Framework of the Waste Metal Ions Detection System

Based method is the basic principle and application of electrochemical experiment means, on the basis of the electrochemical properties is measured in matter content analysis method, are mainly in the detection of basic heavy metal selective electrode method, polarographic analysis, potentiometric stripping and stripping voltammetry that could be listed as the follows, respectively.

- Polarography. Polarography dropping mercury electrode is used to electrolytic solution under test, and based on the curve in the core process of electrolysis for analysis of electrochemical method. Polarography instrument is simple, fast analysis speed, various material measured at the same time, if the bottom is a good choice or bottom liquid has better catalytic material, we can get a higher sensitivity and lower detection limit [7].

- Stripping voltammetry. Through an electrolytic will be measured which electrodeposition on the electrode, and then applying a basic reverse voltage causes concentration on the electrode material to dissolution, according to the given in the dissolution process of volt-ampere curve to conduct quantitative analysis method called stripping voltammetry, is a kind of combined with electrolytic enrichment and determination of dissolution of method.
- Ion selective electrode method. Ion selective electrode is determined by measuring the electrode potential to measure ion activity of electrochemical methods, the ion selective electrode potential and corresponding ion activity in the general solution of logarithm linear relationship. With measuring instrument to measure ion electrode and reference electrode of electromotive force that can calculate the measured the activity of certain ions in the solution components. Ion selective electrode method, the samples can be directly to it from the sample color, turbidity, suspended solids, or the effects of viscosity.

The None Mercury Electrode Electrochemical. Electrochemical method was developed in the recent years the competitive methods of heavy metal wastewater treatment. Wastewater treatment using the electrochemical principle, it has the following advantages. (1) Equipment is small in size as cover an area of less, easy and flexible operation. Therefore the law is called the cleaning process. But electrochemical method also exist, high cost, large energy consumption of the oxygen and hydrogen evolution such as the lack of side reaction. (2) Along with the development of science and technology, the improvement of traditional electrochemical treatment process and the development of the new type of electrochemical reactor, the electrochemical method applied in 
the field of heavy metal wastewater treatment is more effective and more extensive. (3) Without adding any of the oxidant, chemicals such as flocculant and it is treated separately and can be combined with other technologies, improve the wastewater biochemical properties.

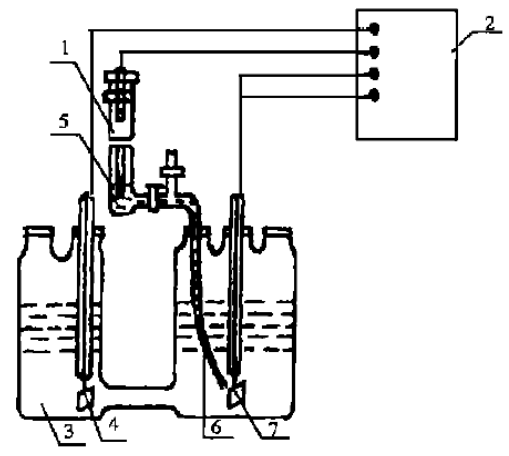

Figure 3. The Demonstration of the Electrode Electrochemical Analysis

Electrochemical oxidation directly is the use of high voltage anode oxidation degradation of the organic or basic inorganic pollutants in wastewater, pollutants in the process of reaction directly with electrodes for electron transfer. In the process of oxidation, pollutants are oxidation degree is not the same. Why some toxic pollutants by oxidation of nonpoisonous pollutants, biochemical treatment of the pollutants was oxidized to biochemical treatment of the material, known as the electrochemical conversion and some of the pollutants are completely for the stability of the inorganic oxide, called oxidation and electrochemical combustion completely [8-9].

After $\mathrm{pH}$ pool will waste water $\mathrm{pH}$ adjustment to the optimal value, again after initial sediment of wastewater pretreatment after pretreatment of waste water into the electrochemical reaction system, continuous processing. Alternating current into a low voltage direct current to provide basic power for electrochemical devices; After electrochemical reaction system treatment of water by gravity from the flocculation in addition to the bubble pool, the flocculation defoaming pool flocculation defoaming and coagulant aid; Entered the second pond of inclined plate and sewage for solid-liquid separation, can satisfy the supernatant fluid, sludge by collecting into sludge pumping station, after concentrated the sludge concentration pool filter again. The primary steps could be organized as the follows. (1) The water of suspended particles, colloidal pollutants under the action of "micro flocculation agent" lost stability because oxidation produced by ion interaction between colloidal particles electric double layer was compressed, at the same time and the counter ion produced by electrolytic ion in the water electricity neutralization makes electrostatic repulsion decreases the adsorption force dominated and make the colloid coagulation effect. (2) Steady pollutant particles and obtain collide with each other, between micro flocculation agent combined into large flocs visible to the naked eye.

\section{Conclusion}

In this paper, we conduct research on none-mercury electrode electrochemical and core applications on waste water metal ions detection under the background of green industry. Electroplating process need to use a lot of heavy metal material, electroplating raw materials were unemployed as a waste, not only pollute the environment, and the high processing cost. The essence of the pollution of the environment resources use is not sufficient, especially heavy metal, raise the utilization rate of heavy metals through online recovery technology is directly reduce the pollution of the environment. The technology is also clean the production reduction, recycling and reuse, the embodiment of the four principles, innocuity make the enterprise from the end of the passive management into active cleaner production. Under the green industry background, we propose the novel perspective on the analysis of the corresponding challenges that is innovative. 


\section{References}

[1] Jugović, Branimir, et al. "Polypyrrole-based Enzyme Electrode with Immobilized Glucose Oxidase for Electrochemical Determination of Glucose." Int. J. Electrochem. Sci 11 (2016): 1152-1161.

[2] Almazán-Ruiz, Francisco J., et al. "Scale-up of rotating cylinder electrode electrochemical reactor for $\mathrm{Cu}$ (II) recovery: Experimental and simulation study in turbulence regimen." Electrochimica Acta 77 (2012): 262-271.

[3] Deng, Yan, et al. "Preparation and electrochemical behavior of L-glutamate electrochemical biosensor." Journal of biomedical nanotechnology 9.2 (2013): 318-321.

[4] Aghazadeh, M., et al. "One-step electrochemical preparation and characterization of nanostructured hydrohausmannite as electrode material for supercapacitors." RSC Advances 6.13 (2016): 10442-10449.

[5] Kaur, Balwinder, and Rajendra Srivastava. "Simultaneous electrochemical determination of nanomolar concentrations of aminophenol isomers using nanocrystalline zirconosilicate modified carbon paste electrode." Electrochimica Acta 141 (2014): 61-71.

[6] $\mathrm{Wu}$, Wenling, et al. "Facile fabrication of polyaniline nanotubes using the self-assembly behavior based on the hydrogen bonding: a mechanistic study and application in high-performance electrochemical supercapacitor electrode." Electrochimica Acta 152 (2015): 126-134.

[7] Zheng, Honghe, et al. "A comprehensive understanding of electrode thickness effects on the electrochemical performances of Li-ion battery cathodes." Electrochimica Acta 71 (2012): 258-265.

[8] Sharma, Vimal, et al. "Electrochemical determination of adenine using a glassy carbon electrode modified with graphene oxide and polyaniline." Microchimica Acta (2016): 1-8.

[9] Singh, Seema, Vimal Chandra Srivastava, and Indra Deo Mall. "Multistep optimization and residue disposal study for electrochemical treatment of textile wastewater using aluminum electrode." International Journal of Chemical Reactor Engineering 11.1 (2013): 31-46. 